Original Research Paper

\title{
Correlation Analysis and Modeling of EEG - EMG Signal for Startle-Induced Seizures
}

\author{
${ }^{1}$ B. Pushpa, ${ }^{2}$ D. Najumnissa and ${ }^{3}$ Dinesh Nayak \\ ${ }^{1,2}$ Department of Electronics and Instrumentation Engineering, B.S. Abdur Rahman University, Chennai, India \\ ${ }^{3}$ Department of Neurology, Fortis Malar Hospital, Adayar, Chennai, India
}

\author{
Article history \\ Received: 25-10-2017 \\ Revised: $12-12-2017$ \\ Accepted: 23-12-2017 \\ Corresponding Author: \\ B. Pushpa \\ Department of Electronics and \\ Instrumentation Engineering, \\ B.S. Abdur Rahman University, \\ Chennai, India \\ E-mail: pushpa_akm@bsauniv.ac.in
}

\section{Introduction}

Epilepsy is a chronic non communicable disorder of the brain that affects people of all age. World Health Organization (WHO) surveys report that about 50 million people (nearly $80 \%$ ) worldwide have epilepsy, and about $70 \%$ of all epileptics are treated appropriately. Accurate diagnosis of the epilepsy is essential for both short term and long term management. The major cause of neurological disorder is seizures. It occurs as a symptom transient, reversible disruption of brain function that is not associated with increased risk of seizure recurrence, such as fever. Accurate diagnosis of the epileptic syndrome in neonates, infants, children, teenagers and adult is very important for their treatment (Evangelia et al., 2016).

EEG is one of the essential tool which gives a useful information about brain function and neurological disorders. To diagnosis the status epilepticus, the minimum standards should be used. A $12-24 \mathrm{~h}$ data is sufficient for reporting the event. Benbadis and Tatum (2003) future, we study the presence of epileptic form activities such as spikes, slow rhythm, and highfrequency epileptic form oscillations. By using epileptic form oscillation we can confirm the presence of epilepsy easily (Indiradevi et al., 2008). In spite of 40 year analysis in to the physiology of epilepsy, it is still impossible to explicate when a seizure occurs and what timeframe is accepted as sensible between the two states - that is, the period of transition from a relatively normal brain state to when the clinical seizure occurs. Seizures encompass a large portion of the cortex, involving thousands of interacting neurons. As a result we can analyse the epileptic brain as a system with important mechanisms from primary seizure (Asha et al., 2013).

Seizure dynamics were investigated using many different mathematical methods, both linear and nonlinear (Pijin et al., 1991) a study has reported that the use of non linear EEG signal and features for the classification of epileptic event or non epileptic event, demonstrated a high degree of accuracy. Due to body movement and cranial muscle activity $(>30 \mathrm{~Hz})$, eye blink and motion artifacts are separated and removed using ICA and Gamma sub band filtering $(30-50 \mathrm{~Hz})$. The predofant frequencies have been quantitatively verified by spectrographic investigation (Schiff et al., 2000). The statistical feature spectral entropy estimation technique is used for analysis, Based on this Separability and Correlation analysis (SEPCOR) they select the optimal feature and ranking the channels based on Variability measure (V-measure). The SEPCOR analyse uses feature vector arranged in a descending order of V- 
measure and a correlation matrix. The features with a correlation highest MAXCORR are included and the feature with lowest V-measure are excluded. These features are applied to Multilayer Perceptron (MPL) and K-Nearest Neighbor (K-NN) has resulted in accuracy 96 and $99.6 \%$ respectively. The performance is tested in different values of correlation threshold. Among this classifier the K-NN performs with less computation time whereas MLP has higher computation time (Padmashri and Sriraam, 2016).

Valderrama et al. (2012) have analysed multiple channel EEG, ECG and IEEG for seizure prediction during the pre-ictal period. The algorithmic features like time domain, frequency domain, time and frequency domain are analysed for in-sample and out-sample classification. Mean represent the frequency distribution of the signal and the feature standard deviation, IQR and variance signify the amount of changes in frequency distribution. Zbilut et al. (2002) entropy quantifies the degree of complexity in a time series. Median, Kurtosis and mode will have specific frequency bands (Evangelia et al., 2016; Zhang et al., 2008). This study uses all the available seizures and data from first half of the seizure is used for testing and training the samples. The correlation coefficient between the features was computed. For EEG features, the highest positive correlation was obtained for mean, skewness, and the relative power in the delta band $(0.1-4 \mathrm{~Hz})$. During the pre-ictal state, Slow-Wave Sleep (SWS) lasts for several minutes before the onset of the seizure, consistent with the increased high-frequency power or decreased lowfrequency power of the heart rate. The features are not unique for the pre-seizure activity. The average pre-ictal period of approximately $30 \mathrm{~min}$ but many were false positive. For seizure prediction, the pre-ictal states do not reflect a deterministic, but pre-ictal state may lead to improved performance for control algorithms.

Electro-Encephalography (EEG) is an inexpensive and an important clinical tool for the evaluation and treatment of neurophysiologic disorders. The study of relationship between EEG and EMG provide us with physiological information about how activities of the cerebral cortex, mainly those of the sensory-motor cortex are related to the movement of interest, whether it is voluntary or involuntary (Lew et al., 2012). In case of voluntary movement, the EEG-EMG correlation is done mainly to investigate mechanisms underlying the central motor control and its disorders. Since the movementrelated cortical electric activities are usually small as compared to the background EEG activity, they cannot be identified by visual inspection of the raw record, even if they might occur in close time relation to the movement (Sylvia et al., 2014).

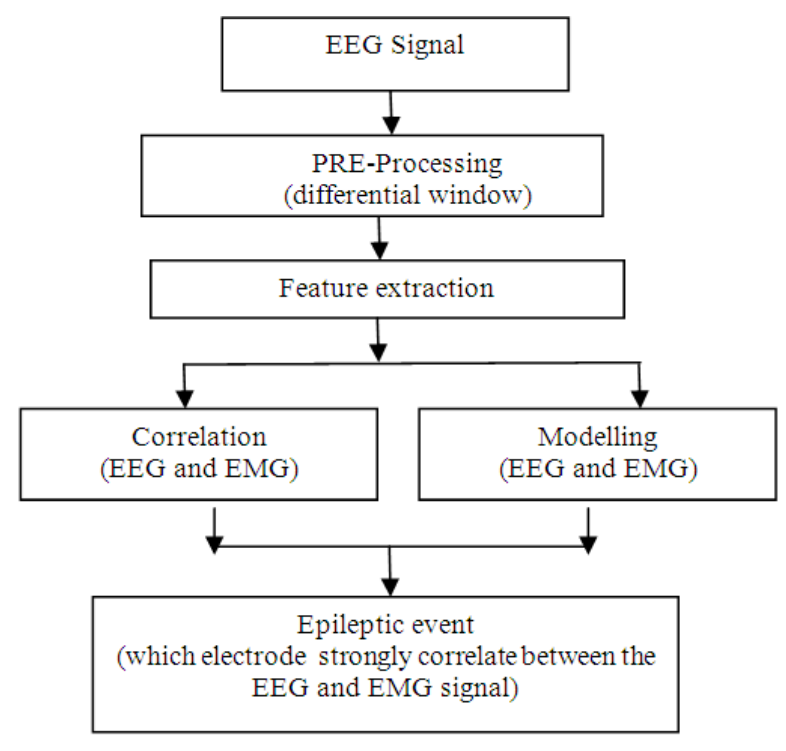

Fig. 1: Schematic sketch of the modeling and correlation

The objective of this study to analyze startle type epilepsy and to find transfer function model, further we are identifying the channel of the ictal onset. The block diagram is shown in the Fig. 1.

\section{Materials and Methods}

\section{Subjects}

In this study, a long term has been evaluated for EEG and EMG signal. The Fig. 2 shows an example of Startle type Epilepsy. The EEG data is acquired from 10 subjects with startle type epilepsy (4 male and 6 female; age range 1-16 years), who underwent long-term video EEG montoring at Fortis Malar Hospital, Adyar, Chennai. The EEG signal were recorded through a digital video-EEG system (20 channel EEG system , Nicolet One Neuro diagnostic system, ) from electrode (Fp1, F3, C3, P3, O1, Fp2, F4, C4, P4, O2, F7, T1, T3, T5, F8, T2, T4, T8, T6, Fz, Cz, Pz, ECG, EMGrl, EMGll,) attached using electrodes 10-20 system of electrode placement. Jayant et al. (2016) a bipolar electrode montage is used in the analysis. Each electrode output is band pass filtered by $0.5-100 \mathrm{~Hz}$ during recording by setting the low cut and high cut at $0.3 \mathrm{~Hz}$ and $70 \mathrm{~Hz}$, respectively. The EEG data were subsequently digitized through a 12-bit A/D converter with a sampling rate at $256 \mathrm{~Hz}$ and stored in the hard disk of PC for off line analysis. A data set containing these events were then pruned from the main file and stored as ASCII file. We analyzed the EEG activity of these electrodes $\mathrm{O}_{1}, \mathrm{O}_{2}, \mathrm{~T}_{2}, \mathrm{~T}_{3}, \mathrm{~T}_{4}, \mathrm{~T}_{6}, \mathrm{~F}_{\mathrm{z}}, \mathrm{P}_{\mathrm{z}}, \mathrm{C}_{\mathrm{z}}$ and ECG which represent Frontal (F), Central (C), Partial (P), Temporal $(\mathrm{T})$ and Occipital $(\mathrm{O})$ area of the brain. 


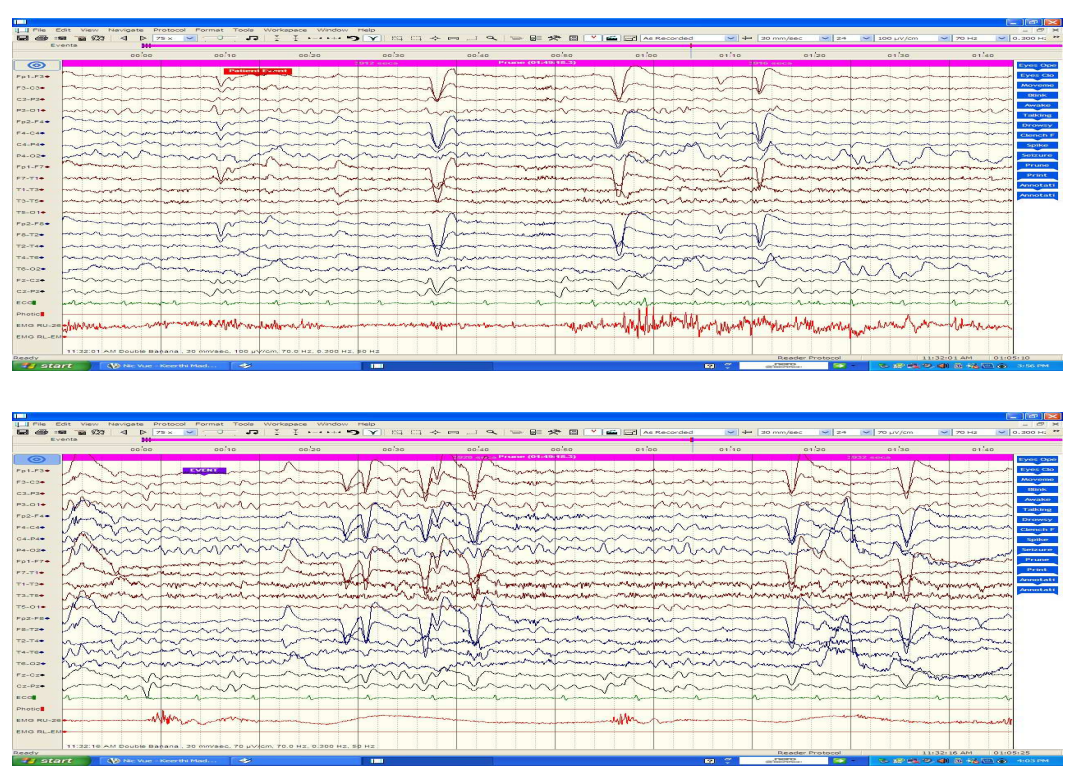

Fig. 2: An example startle-induced seizures

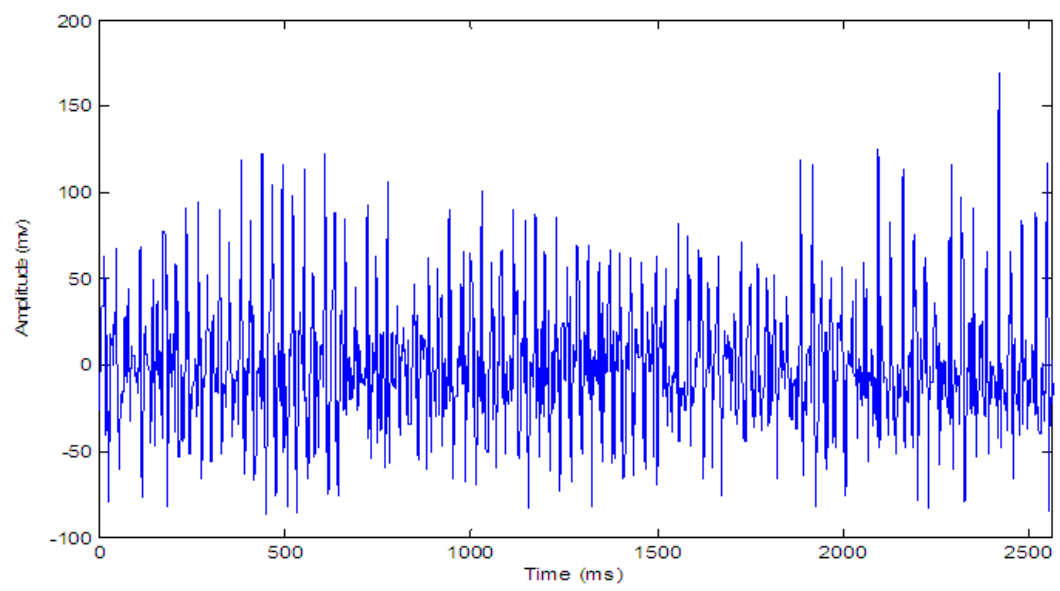

(a)

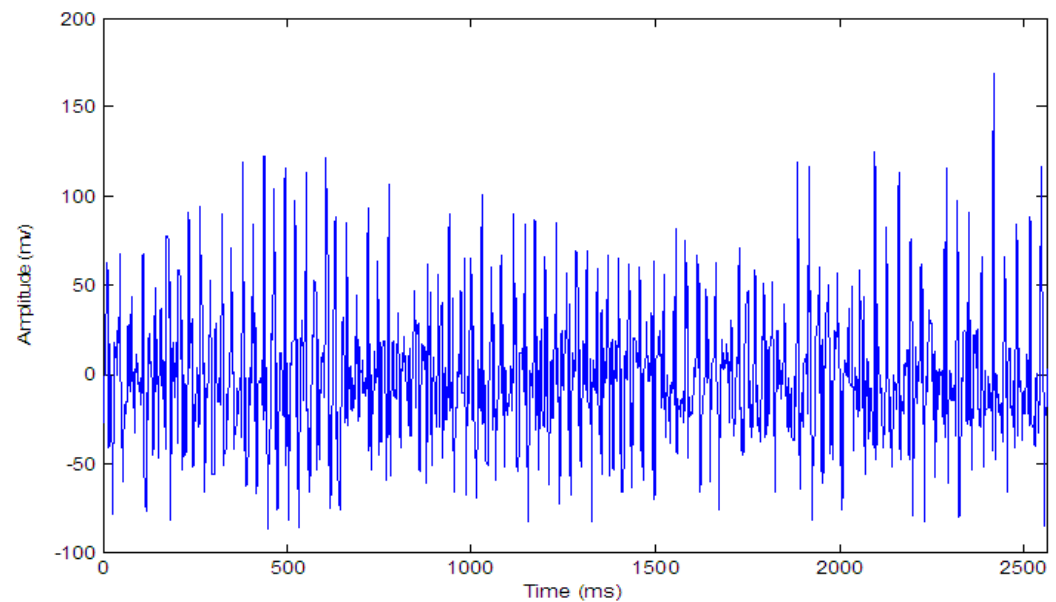

(b)

Fig. 3: (a) EEG signal (b) Differential windowed EEG signal 
Along with this, EMG signal from right and left tebilian muscles are also considered to find the epileptic event. Indiradevi et al. (2008) the block diagram as shown in Fig. 2 represents for the startle type epilepsy.

\section{Pre-Processing}

The major difficulty in EEG is artifacts removal in the signals. All data were pre-processed with a notch filter at $50 \mathrm{~Hz}$ for power line disturbance rejection, and with band pass filter $(1.0-80.0 \mathrm{~Hz})$ for environment noise reduction (Padmashri and Sriraam, 2016). A Differential Window (DW) (Parvez and Paul, 2016) is used in differentiating values between preictal/ictal EEG signal. It is observed that the signals are rather more distinctive. After applying the DW to the data of EEG signal and EMG signal it discriminates between the pre-ictal and ictal states better. Majumdar and Vardhan (2011) the Fig. $3 a$ and $b$ shows the EEG signal and pre-processed signal.

\section{Feature Extraction}

After preprocessing, the statistical features are extracted for $10 \mathrm{~s}$ epoch. In 24- channel data, the features extracted are Inter Quartile Range (IQR), variance, mean, median, mode, skewness, kurtosis, entropy, Mean Absolute Deviation (MAD) and standard deviation extracted for selected electrodes. Kurtosis and skewness are measures of 'peakedness' and 'asymmetry' respectively. Karoly et al. (2016) the Inter Quartile Range (IQR) is a measure of variability, based on dividing a data set in to quartiles. Bedeeuzzamana et al. (2012) the Median Absolute Deviation (MAD) is a robust measure of the variability of a univariate sample of quantitative data. Bedeeuzzamana et al. (2012; Helen et al., 2013).

\section{Correlation}

From the extracted feature set a correlation coefficient ( $\mathrm{r}$ ) is computed between the central line electrodes like $F_{z}, P_{z}, C_{z}, \mathrm{O}_{1}, O_{2}, T_{2}, T_{3}, T_{4}$ and EMG right leg and left leg electrodes for each subject using linear fit technique. The correlation coefficient $r$ is given by Equation (1):

$$
r=\frac{\sum_{i=1}^{n}\left(X_{i}-\bar{X}\right)\left(Y_{i}-\bar{Y}\right)}{\sqrt{\sum_{i=1}^{n}\left(X_{i}-\bar{X}\right)^{2}} \sqrt{\sum_{i=1}^{n}\left(Y_{i}-\bar{Y}\right)^{2}}}
$$

where, $\bar{X}=\left(\sum_{i-1}^{n} X_{i}\right) / n, \& \bar{Y}=\left(\sum_{i-1}^{n} Y_{i}\right) / n, X$ and $Y X$ and $Y$ are the feature values of EEG and EMG electrode (Ravindra and Ramakrishnan, 2014).

\section{Modeling of EEG and EMG}

Modeling of EEG and EMG signal is to understand the complexity of the brain with the muscular activity (Kasabov and Elisa, 2015). In this section, the time series data were used to model the multichannel EEG and EMG right and left leg. Gatein et al. (2013) modeling is the abstraction of a real process to characterize its behavior. The aim of Modeling is to enhance the investigation of phenomena in order understand the cause-effect relationships (Yang et al., 2015). The set of processes in a system determines the behavior of the system. Every process is determined by its physical and chemical properties, which are not always easily known. A model tries to emulate the 'essential aspects' of the system behavior, simplified by choosing the most significant properties. Garthwaite et al. (1988) modeling complex projects. Wiley, New Jersey) so, a model based technique is used on data without having previous knowledge of the system. The model describes how the outputs depend on the inputs. System identification tries to estimate a black or grey model of a dynamic system. Tukey (1960) some examples of identification aims could be listed here:

- To analyze the properties of the system

- To forecast the evolution of the system

- To identify the interaction between coupled systems

- To improve the internal knowledge of the system

Time series analysis definition was given by (Tukey, 1960) "Time series analysis consists of all the techniques that, when applied to time series data, yield, at least sometimes, either insight or knowledge, and everything that helps us choose or understand these procedures". Thus, a time series can expose some concealed information about the system, as periodicity, outliers and trends, using typical statistics estimators (Diwaker et al., 2016).

\section{Results and Discussion}

In this study we have shown the consistency of processing the seizure event, by correlating the EEG and EMG signal. Ten second epoch were taken and features were extracted. Ictal EEG channels shows an initial midline vertex discharge followed by diffuse attenuation or low voltage fast activity, which may have onset areas of structural brain abnormality the Table 1 shows the features extracted from the electrodes Fz, Pz, Cz, O1, O2, T2, T3, T4,T6, ECG, EMG right and left leg. The feature of the electrode Fz, Pz, Cz, O1, O2, T2, T3, T4, T6, ECG is correlated with EMGRL and EMGLL is shown in Table 2. 
Table 1: Feature extraction of electrode Features

\begin{tabular}{|c|c|c|c|c|c|c|c|c|c|c|}
\hline Electrode & Mean & IQR & MAD & Median & Mode & Variance & $\begin{array}{l}\text { Standard } \\
\text { deviation }\end{array}$ & Entropy & Kurtosis & Skewness \\
\hline$\overline{\mathrm{Fz}}$ & 12.1000 & 73.300 & 35.760 & 10.850 & -17.010 & 1773.360 & 40.800 & 1.0330 & 1.8820 & 0.09700 \\
\hline $\mathrm{Pz}$ & -13.4000 & 72.300 & 37.840 & -13.530 & -31.722 & $2085.310 \mathrm{a}$ & 44.470 & 1.0227 & 2.1910 & -0.02240 \\
\hline $\mathrm{Cz}$ & 13.5600 & 69.830 & 35.822 & 12.863 & -7.911 & 1841.020 & 41.710 & 1.0286 & 2.1035 & 0.02600 \\
\hline $\mathrm{O} 1$ & -3.9843 & 73.499 & 38.441 & -4.590 & -26.394 & 2169.700 & 45.253 & 1.0420 & 2.2144 & 0.02770 \\
\hline $\mathrm{O} 2$ & -2.9160 & 68.670 & 36.584 & -3.385 & -21.244 & 2119.550 & 43.400 & 1.0450 & 2.2820 & -0.00270 \\
\hline $\mathrm{T} 2$ & 22.7450 & 70.620 & 36.319 & 22.300 & 2.352 & 1892.600 & 42.291 & 0.9740 & 2.0950 & 0.00320 \\
\hline T3 & 7.7480 & 71.796 & 37.097 & 7.212 & -13.770 & 1986.553 & 43.316 & 1.0410 & 2.1370 & 0.01560 \\
\hline $\mathrm{T} 4$ & 11.4750 & 71.693 & 37.016 & 11.082 & -10.060 & 1978.900 & 43.228 & 1.0334 & 2.1336 & 0.00174 \\
\hline T6 & -3.9920 & 69.530 & 36.556 & -4.261 & -22.056 & 1970.420 & 43.058 & 1.0453 & 2.2250 & -0.00860 \\
\hline ECG & 7.4800 & 59.540 & 43.789 & 17.255 & 14.612 & 4694.120 & 66.788 & 0.9458 & 13.2370 & -1.95300 \\
\hline EMGRL & 17.4950 & 111.625 & 111.997 & -11.221 & -40.232 & 52361.450 & 227.240 & 1.0216 & 22.5750 & 4.02600 \\
\hline EMGLL & 6.1670 & 116.110 & 111.830 & -22.165 & -50.834 & 44170.200 & 227.450 & 1.3314 & 22.5760 & 4.02000 \\
\hline
\end{tabular}

Table 2: Correlation between features

\begin{tabular}{|c|c|c|c|c|c|c|c|c|c|c|c|}
\hline $\begin{array}{l}\text { EEG-EMG } \\
\text { electrode }\end{array}$ & Seizure & Mean & IQR & MAD & MIN & MAX & VAR & STD & Entropy & Kurtosis & Skewness \\
\hline Fz-EMG RL & No event & 0.14750 & 0.03230 & -0.01050 & 0.10050 & 0.1952 & -0.0202 & $3.06 \mathrm{E}-04$ & -0.0048 & 0.0795 & 0.1334 \\
\hline Pz-EMG RL & & 0.04750 & -0.10430 & -0.02020 & 0.08320 & -0.0983 & -0.1719 & -0.04050 & 0.0153 & 0.0409 & 0.0465 \\
\hline Cz-EMG RL & & 0.03920 & -0.01690 & 0.05220 & -0.01540 & -0.2468 & 0.0348 & 0.04850 & 0.0144 & 0.0591 & 0.0605 \\
\hline O1-EMG RL & & 0.03600 & 0.25800 & 0.36940 & -0.16540 & -0.2354 & -0.3647 & -0.13650 & 0.1256 & & 0.2658 \\
\hline O2-EMG RL & & 0.13560 & 0.02500 & & 0.12940 & & & & & & 0.1250 \\
\hline T2-EMG RL & & 0.03500 & 0.14500 & 0.03600 & 0.02140 & 0.0365 & & 0.24510 & & & 0.1478 \\
\hline T3-EMG RL & & 0.12540 & 0.02140 & 0.03610 & 0.12340 & & & 0.02140 & & & 0.0235 \\
\hline T4-EMG RL & & 0.12650 & & & & & & & & & \\
\hline T6-EMG RL & & & & & & & & & & & \\
\hline ECG-EMG RL & & & & & & & & & & & \\
\hline Fz-EMG LL & & & & & & & & & & & \\
\hline Pz-EMG LL & & & & & & & & & & & \\
\hline $\mathrm{Cz}-\mathrm{E}$ & & & & & & & & & & & \\
\hline G LL & & & & & & & & & & & \\
\hline G LL & & & & & & & & & & & \\
\hline S LL & & & & & & & & & & & \\
\hline G LL & & & & & & -0.36 & & & & & \\
\hline J LL & & & & & & & & & & & \\
\hline $\mathrm{J} \mathrm{LL}$ & & & & & & & & & & & \\
\hline MG LL & & & & & & & & & & & 0.0 \\
\hline Fz-EMG RL & During event & & & & & & & & & & \\
\hline Pz-EMG RL & & 0.154 & & & & 30 & & & & & 36 \\
\hline Cz-EMG RL & & 0.07840 & 0.84200 & & & & & 30 & & & 0.8147 \\
\hline IG RL & & 0.12500 & 0.24100 & 690 & 50 & 0.3215 & & & 0.0214 & 45 & 0.1262 \\
\hline IG RL & & 0.23140 & 0.15620 & 0.32540 & 0.14520 & 0.3652 & & & & & 0.2154 \\
\hline G RL & & 0.23650 & 0.14590 & 0.32540 & & 0.1265 & & & & & 0.1586 \\
\hline T3-EMG RL & & 36920 & & & 0.12650 & 0.1235 & & & & 0.2456 & 0.1234 \\
\hline T4-EMG RL & & 0.23650 & & 0.12350 & 0.25430 & 0.2136 & 0.2365 & & 0.0236 & 0.2365 & 0.01523 \\
\hline T6-EMG RL & & 0.36210 & 0.12360 & 0.25420 & 0.12540 & 0.0365 & 0.0125 & 0.12650 & 0.0123 & 0.3214 & 0.0214 \\
\hline ECG -EMG RL & & 0.01230 & & & & & 0.1236 & 0.01530 & 0.0236 & 0.0125 & 0.3214 \\
\hline Fz-EMG LL & & 0.01230 & 0.01240 & 0.10230 & 0.01450 & 0.0265 & 0.2150 & 0.01230 & 0.0153 & 0.0123 & 0.1236 \\
\hline Pz-EMG LL & & 0.32150 & 0.12450 & 0.01234 & 0.01230 & 0.0265 & 0.0145 & 0.03652 & 0.0125 & 0.0156 & 0.0231 \\
\hline Cz-EMG LL & & & & & & & & & & & \\
\hline O1-EMG LL & & 0 & & & & & 0.2 & & & 0.2 & 0.3652 \\
\hline O2-EMG LL & & & & & & & & & & & 0.3652 \\
\hline T2-EMG LL & & & & & & & & & & & \\
\hline T3-EMG LL & & & & & & & & & & & \\
\hline T4-EMG LL & & & & & & & & & & & \\
\hline T6-EMG LL & & & & & & & & & & & 0.3241 \\
\hline ECG-EMG LL & & 0.12540 & 0.36520 & 0.12560 & 0.25410 & 0.1258 & 0.2154 & 0.12650 & 0.1236 & 0.2548 & 0.3215 \\
\hline
\end{tabular}


Table 3: Gain value of time series data

\begin{tabular}{lll}
\hline Eeg & Emgrl & Emgll \\
\hline $\mathrm{Fz}$ & 1.388 & 0.4426 \\
$\mathrm{Pz}$ & 1.324 & -0.4507 \\
$\mathrm{Cz}$ & 1.041 & 2.8800 \\
\hline
\end{tabular}

From Table 1, it is seen that the features are extracted for the central line electrodes, occipital lobe, temporal lobe, ECG and EMG. Table 2 shows the correlation between the extracted features of EEG and EMG. It can be inferred that there is poor correlation between the electrodes O1, O2, T2, T3, T4, T6, ECG with EMGRL and EMGLL. A wide range of variation is given, However there is a strong correlation between the electrodes $\mathrm{Fz}, \mathrm{Pz}$, and $\mathrm{Cz}$ with EMGRL.

The Table 3 shows that the modeling of time - series EEG and EMG signal, from this we can validate that the correlation between electrodes and modeling of the time series data. System Identification tool box is used to obtain the first order transfer function. From the transfer function the gain is calculated and is shown in Table 3 . The RGA is used to pair the EEG and EMG signal for right leg and left leg. The RGA for time series data EEG and EMGRL and EMGLL is Equation 2, 3 and 4.

From this the $R 1$ shows the interaction between the electrodes Fz, Pz EMGRL and EMGLL. The array gives the maximum value for right leg when compared to the left leg. $R 2$ shows the interaction between electrode $\mathrm{Pz}$, $\mathrm{Cz}$, EMGRL and EMGLL. From this array value, the right leg as maximum value. $R 3$ shows the interaction between electrode $\mathrm{Fz}, \mathrm{Cz}, \mathrm{EMGRL}$ and EMGLL. In this we have maximum value for the right leg and minimum value for the left leg. Since $\mathrm{Cz}$ electrodes will have sleep waves, so the interaction during the epileptic event show the minimum value.

Relative Gain Array of time series data:

$$
\begin{aligned}
& R 1=\left[\begin{array}{ll}
0.5163 & 0.4837 \\
0.4837 & 0.5163
\end{array}\right] \\
& R 2=\left[\begin{array}{ll}
0.8904 & 0.1096 \\
0.1096 & 0.8904
\end{array}\right] \\
& R 3=\left[\begin{array}{cc}
1.1303 & -0.1303 \\
-0.1303 & 1.1303
\end{array}\right]
\end{aligned}
$$

\section{Conclusion}

It is also noticed that there are conflicting results about EEG and EMG. There is a strong correlation between the central line electrode and EMGRL for entire regions in the brain, whereas other electrodes show no correlation between the EMGRL and EMGLL. From the modeling of time series data, during the event the electrode $\mathrm{Fz}$ and $\mathrm{Pz}$ shows the high gain value and remaining electrode shows the low and negative gain value. The possible relation between the gain value can be validated by RGA. In RGA the array value shows that the maximum value for the electrode $\mathrm{Fz}, \mathrm{Pz}, \mathrm{Cz}$ and EMGRL and lowest value for the EMGLL. So that we conclude that there are significant variation in EEG electrode and EMG electrode during the epileptic event. Although the central line will pick up slow waves, during the occurrence of the event, it shows correlation between EMG signals. From this we conclude that for startle type epilepsy these electrodes Fz, Pz \& Cz show the abnormalities in the brain.

\section{Author's Contributions}

B. Pushpa: This work was carried out in collaboration between all authors. The author assisted all the steps of this work. She designed the study, assisted sample collection, data analysis and manuscript preparation.

D. Najumnissa: Supervised the study, coordinated the data-analysis and revised the manuscript.

Dinesh Nayak: Contribution of data and Interpretation of data.

\section{Ethics}

This article is original and contains unpublished material. The corresponding author confirms that all of other authors have read and approved the manuscript and no ethical issues involved.

\section{References}

Yang, A., D.H. Arndt, R.A. Berg, J.L. Carpenter and K.E. Chapman et al., 2015. Development and validation of a seizure prediction model in critically ill children. Seizure, 25: 104-111.

Asha, A., C. Sudalaimani, P. Devanand, T.T. Elizabeth and S. Sudhamony, 2013. Automated seizure detection from multichannel EEG signals using support vector machine and artificial neural networks. Proceedings of the International MultiConference on Automation, Computing, Communication, Control and Compressed Sensing, Mar. 22-23, IEEE Xplore Press, Kottayam, India. DOI: 10.1109/iMac4s.2013.6526473

Benbadis, S.R. and W.O. Tatum, 2003. Overinterpretation of EEGs and misdiagnosis of epilepsy. J. Clin. Neurophysiol., 20: 42-44.

Lew, E., R. Chavarriaga, S. Silvoni and J.D.R. Millán1, 2012. Detection of self-paced reaching movement intention from EEG signals. Frontier Neuro Eng. DOI: $10.3389 /$ fneng.2012.00013 
Evangelia, P., I. Evangelia, I.M. Zacharaki and K. Michael, 2016. Improving classification of epileptic and non-epileptic EEG events by feature selection. J. Neurocomputing.

DOI: 10.1016/j.neucom.2015.06.071

Gatein, H., L. Benjamin, P.V. Bogaert, F. Grenez and A. Nonclercq, 2013. Early detection of epileptic seizures based on parameter identification of neural mass model. Comput. Biol. Med., 43: 1773-1782.

Helen, T.O., K.A.I. Aboalayon and M. Faezipour, 2013. Efficient EEG analysis for seizure monitoring in epileptic patients. Proceedings of the IEEE Long Island Systems, Applications and Technology Conference, May 3-3, IEEE Xplore Press, Farmingdale. DOI: 10.1109/LISAT.2013.6578218

Zbilut, J.P., N. Thomassona and C.L. Webberb, 2002. Recurrence quantification analysis as a tool for nonlinear exploration of nonstationary cardiac signals. Med. Eng. Phys., 24: 53-60.

DOI: $10.1016 / \mathrm{S} 1350-4533(01) 00112-6$

Jayant, N.A., A. Hani, P. Thirumala and T.N. Tsuchida, 2016. American clinical neurophysiology society guideline 3: A proposal for standard montages to be used in clinical EEG. J. Clin. Neurophysiol., 33: 312-316. DOI: 10.1097/WNP.0000000000000317

Majumdar, K.K. and P. Vardhan, 2011. Automatic seizure detection in Ecog by differential operator and windowed variance. IEEE Transt. Neural Syst. Rehabilitation Eng., 20: 749-755.

DOI: 10.1109/TNSRE.2011.2157525

Indiradevi , K.P., E. Elias, P.S. Sathidevi, S.D. Nayak and K.R. Krishnan, 2008. A multi-level wavelet approach for automatic detection of epileptic spikes in the electroencephalogram. Comput. Biol. Med., 38: 805-816. DOI: 10.1016/j.compbiomed.2008.04.010

Bedeeuzzamana, M., T. Fathimab, Y.U. Khanb and Omar Farooq, 2012. Seizure prediction using statistical dispersion measures of intracranial EEG. J. Biomedical Signal Proc. Control, 10: 338-341. DOI: $10.1016 /$ j.bspc.2012.12.001

Valderrama, M., C. Alvaradoa, S. Nikolopoulosa, J. Martineriea and C. Adama et al., 2012. Identifying an increased risk of epileptic seizures using a multifeature EEG-ECG classification. Elsevier J. Biomedical Signal Proc. Control, 7: 237-244. DOI: $10.1016 /$ j.bspc.2011.05.005

Parvez, M.Z. and M. Paul, 2016. Epileptic seizure prediction by exploiting spatiotemporal relationship of EEG signals using phase correlation. IEEE Trans. Neural Syst. Rehabilitation, 24: 158-168.

DOI: 10.1109/TNSRE.2015.2458982
Kasabov, N. and C. Elisa, 2015. Piking neural network methodology for modelling, classification and understanding of EEG spatio-temporal data measuring cognitive processes. Inf. Sci., 294: 565-575. DOI: 10.1016/j.ins.2014.06.028

Karoly, P.J., D.R. Freestone, R. Boston, D.B. Grayden and D. Himes et al., 2016. Interictal spikes and epileptic seizures: Their relationship and underlying rhythmicity. Brain, 139: 1066-78. DOI: 10.1093/brain/aww019

Pijin, J.P., J. Van Neerven, A. Noest and F.H. Lopes da Silva, 1991. Chaos or noise in EEG signals; dependence on state and brain site. Electroencephalogy Clin. Neurophysiol., 79: 371-81. PMID: 1718710

Ravindra, B.P. and S. Ramakrishnan, 2014. Analysis of sub-anatomic diffusion tensor imaging indices in white matter regions of Alzheimer with MMSE score. Comput. Methods Programs Biomed., 117: 13-19. DOI: $10.1016 /$ j.cmpb.2014.06.004

Diwaker, S., S.K. Gupta and N. Gupta, 2016. Classification of EEG signal using correlation coefficient among channels as features extraction method. Indian J. Sci. Technol. DOI: 10.17485/ijst/2016/v9i32/100742

Schiff, S.J., D. Colella, G.M. Jacyna, E. Hughes and J.W. Creekmore et al., 2000. Brain Chirps: Spectrographic signatures of epileptic seizure. Clin. Neurophysiol., 111: 953-8. PMID: 10825700

Sylvia, K., S. Ubbink, J. Vles, A.D. Louw and M.D. van Hall et al., 2014. Noninvasive treatment alternative for intractable startle epilepsy. Epilepsy Behavior Case Reports, 2: 49-53 DOI: 10.1016/j.ebcr.2014.02.002

Padmashri, T.K. and N. Sriraam, 2016. Spectral entropy feature subset selection using SECOR to detect alocholic impact on gamma Subband visual event related potentials of multichannel Electroenecphalograms (EEG). Applied Soft Comput., 46: 441-451. DOI: 10.1016/j.asoc.2016.04.041

Tukey, J., 1960. A Survey of Sampling from Contaminated Distributions. In: Contributions to Probability and Statistics: Essays in Honor of Harold Hotelling, Olkin, I., (Ed.), Stanford University Press, Stanford, pp: 448-485.

Garthwaite, J., S.L. Charles and R. Chess-Williams, 1988. Endothelium-derived relaxing factor release on activation of NMDA receptors suggests role as intercellular messenger in the brain. Nature, 336: 385-388. 